\title{
Extraction of Usefulness Factors of Reviews by Factor Analysis
}

\author{
Kenjo Yasui, Fumiko Harada, and Hiromitsu Shimakawa
}

\begin{abstract}
Customers buy products without watching and using products directly in online-shopping. Customers cannot know product's details. Customers can make mistake in selecting products and regret this selection. Our research aims to extract useful information for deciding products customers buy in product review of online-shopping websites. We can prevent customers' regret by using this information. In this paper, we discover common factors between useful reviews for deciding products customers buy in online-shopping. We use factor analyses of actual reviews to discover common factors. We make the dataset for factor analyses in an experiment. The dataset is the set of critical sentences of actual reviews and their scores to evaluate their characteristics, which the examinees give. In this result, we can extract the common factors by which the useful reviews can be derived with the precision over $50 \%$. The common factors can be used for extracting useful information in reviews. Customers can shop without regrets in online-shopping by showing this information.
\end{abstract}

Index Terms-Review, online-shopping, factor analysis, extraction of factors, precision.

\section{INTRODUCTION}

Recently, online-shopping market is growing up under the influence of spreading of WWW. Online-shopping is shopping in WWW. Online-shopping can be possible without going to real shops. Customers buy products without watching and using products directly in online-shopping. Customers cannot know product's sense of use. Customers read reviews to avoid this problem. According to the report from iResearch China Internet Research Center in 2003, review tends to be more important factor than producer and brand for deciding products [1]. It may be because product reviews bring useful information to customers in selecting products since reviews were provided by the past customers who have used the products actually and have some knowledge on the products. However, all of reviews cannot be useful information for deciding products, because reviews are composed by subjective contents, such as impression or estimation. In order to achieve this, common factors between useful reviews for deciding products customers buy is needed for extracting useful information by computers. For example, we suppose there is a factor "information including number" as a common factor between useful reviews for deciding products customers buy. Computers can extract useful

Manuscript received August 15, 2013; revised October 15, 2013

Kenjo Yasui is with the Graduate School of Science and Engineering Ritsumeikan University, Shiga, Japan (e-mail: kenjo@ de.is.ritsumei.ac.jp).

Fumiko Harada and Hiromitsu Shimakawa are with College of Information Science and Engineering, Shiga, Japan (e-mail: harada@cs.ritsumei.ac.jp, simakawa@cs.ritsumei.ac.jp). reviews for deciding products customers buy according to the factor "information including number". For example, a review including the information "It is so heavy for me that the weight is $256 \mathrm{~g}$ " can be extracted.

In this paper, we discover common factors between useful reviews for deciding products customers buy in online-shopping by analyzing actual reviews. Common factors can be used for extracting useful information in reviews by computers. Customers can shop without regrets in online-shopping by this information computers showed.

\section{RELATED WORK}

There are some researches to classify reviews based on some standards. Some researches use whether reviews are positive or negative points for these standards [2]. These positive and negative points are decided by reviews' writers. The research in [3] extracts only a customer's opinion in reviews. This research aims to extract a set of < Subject, Attributes, Value> in reviews, for example <laptop, keyboard, light $>$. There is a research in [4] that observes what attributes of reviews have an impact on e-commerce's sales. This research focuses on reviews' attributes such as readability, degree of including subjective contents, and so on. This research examines how their attributes have an impact on e-commerce's sales. This related research deals with whether reviews have an impact on e-commerce's sales or not. However, it is necessary for customers to focus on customers' opinion to reviews. In that case, we can examine factors deciding products customers buy. By focusing on customers' evaluation to reviews, we can classify reviews by the points of what information customers need.

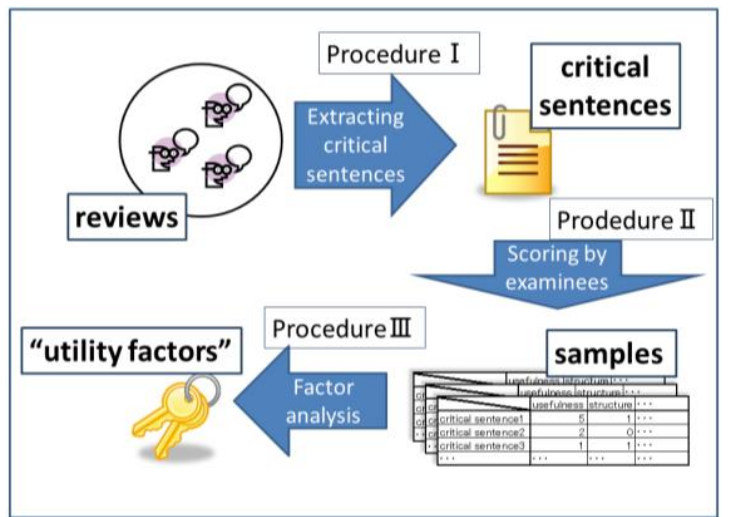

Fig. 1. The flow of the method extracting "utility factors" from reviews.

\section{The METHOD EXTRACTING "UTILITY FACTORS" FOR REVIEWS}

In this research, we extract the common factors between 
useful information on reviews from the reviews in an actual online-shopping website. Usefulness means helpful for customers to choose products in online-shopping. These factors are referred to as "utility factors". Fig. 1 shows the flow of the method extracting "utility factors" from existing reviews. The method extracting "utility factors" is composed of Procedure I, Procedure II, and Procedure III. Procedure I makes the set of critical sentences from reviews for extraction of "utility factors". In Procedure II, the set of samples for analysis are generated through scoring of each critical sentence by each examinee. Procedure III derives "utility factors" from the set of samples by factor analyses. We will explain these procedures in detail in this section.

In this research, we select laptop reviews to extract "utility factors" because we consider laptop reviews is the best for extracting "utility factors". This consideration is based on the fact university students of 20s need laptop computers.

\section{A. Procedure I: Extraction of Critical Sentences}

In Procedure I, we extract critical sentences from the reviews. Extraction of critical sentences is performed individual review. In this research, a sentence including a pair of an item written in the specification table of the product and an adjective is referred to as a critical sentence. For example, if a review includes the sentence "This laptop is so easy to carry because the weight is so light", it is extracted as a critical one, where the words "weight" and "so light" are the item written in the specification table and the adjective, respectively. The set of all of the extracted critical sentences from all reviews is the data for analysis for extraction of "utility factors". The reason why we use only critical sentences for extraction of "utility factors" instead of the entire review is as follows. A review can include the information except impressions and remarks for the corresponding product, which we consider is not useful for customers. We aim to extract the parts including only impressions and remarks by deriving the critical sentences from a review.

In this research, critical sentences are extracted automatically by the computer program. In the first step, this program divides reviews into every sentence by the end characters. In this research, ".", "?", and "!" are selected as the end characters. In the second step, this program finds the sentences including the items in the specification table and adjectives from all sentences extracted in the first step by using the morphological analysis software Mecab [5]. These sentences are referred to as the critical sentences.

\section{B. Procedure II: Making Samples by Examinees' Scoring}

In Procedure II, samples are generated used in analysis for extracting "utility factors". Samples are made by conducting the experiment in which each examinee scores every critical sentence based on some scoring items. In this research, a set of a critical sentence and its scores which an examinee gave in the experiment is referred to as a sample.

\section{1) Setting the scoring items}

We set the scoring items based on the result of a preliminary experiment before the experiment making samples. The scoring items are used to score a critical sentence for an examinee when the experiment making samples conducted. Table I shows the set of scoring items obtained from the result of the preliminary experiment. We guessed 9 of the scoring items expect "Usefulness" associate with "utility factors". The detail of the preliminary experiment is as follows. The examinees of the preliminary experiment are 3 university students in an IT department of $20 \mathrm{~s}$. Each examinee has assumed the scene he buys a laptop computer in an online-shopping website. In this experiment, we used all of the critical sentences of all of the reviews for laptop computer products in "Rakuten dataset" [6] provided by Rakuten Ichiba [7] as actual review. We presented from 100 to 200 critical sentences to each examinee. Under the above assumption, each examinee has scored each presented critical sentences based on the following 2 scoring items.

TABLE I: SCORING ITEMS AND THEIR EXPLANATIONS

\begin{tabular}{|c|c|}
\hline Scoring item & Explanations \\
\hline Usefulness & $\begin{array}{l}\text { Whether the critical sentence lets the } \\
\text { examinee imagine sense of use of products or } \\
\text { not. }\end{array}$ \\
\hline Structure of sentence & $\begin{array}{l}\text { Whether the critical sentence is steady } \\
\text { logically such that readers understand easily } \\
\text { or not. }\end{array}$ \\
\hline Adjective & $\begin{array}{l}\text { Whether there is an adjective in the last of the } \\
\text { critical sentence or not. }\end{array}$ \\
\hline Subjective/Objective & $\begin{array}{l}\text { Whether the critical sentence is subjective or } \\
\text { objective. }\end{array}$ \\
\hline Number & $\begin{array}{l}\text { Whether there are some numbers to show the } \\
\text { product's performance or not. }\end{array}$ \\
\hline Positive/Negative & $\begin{array}{l}\text { Whether the critical sentence is positive or } \\
\text { negative for products. }\end{array}$ \\
\hline New knowledge & $\begin{array}{l}\text { Whether the critical sentence gives new } \\
\text { knowledge to the examinee or not. }\end{array}$ \\
\hline Comparative & $\begin{array}{l}\text { Whether there is a comparative expression } \\
\text { with other products in the critical sentence or } \\
\text { not. }\end{array}$ \\
\hline Specific/Abstract & $\begin{array}{l}\text { Whether the critical sentence is specific or } \\
\text { abstract. }\end{array}$ \\
\hline
\end{tabular}

TABLE II: SCORING ITEMS AND THEIR ANSWER CHOICES

\begin{tabular}{|c|c|}
\hline Scoring items & Scores \\
\hline Usefulness & $\begin{array}{l}\text { Can imagine very well:5, can imagine: } 4, \\
\text { cannot judge:3, cannot imagine very well: } 2 \text {, } \\
\text { cannot imagine: } 1\end{array}$ \\
\hline Structure of sentence & Very steady: 2 , steady: 1 , not steady:0 \\
\hline Adjective & $\begin{array}{l}\text { There is an adjective at the last of the sentence: } 1 \text {, } \\
\text { There is no adjective in the sentence: } 0 \text {, } \\
\text { There is an adjective except the last of the } \\
\text { sentence:- } 1\end{array}$ \\
\hline Subjective/Objective & Subjective:1, Neutral:0, Objective:-1 \\
\hline Number & $\begin{array}{l}\text { There is numbers in the critical sentence: } 1 \text {, } \\
\text { There is not numbers in the critical sentence: } 0\end{array}$ \\
\hline Positive/Negative & Positive:1, Neutral:0, Negative:-1 \\
\hline New knowledge & $\begin{array}{l}\text { There is a new knowledge in the critical } \\
\text { sentence: } 1 \text {, } \\
\text { There is not a new knowledge in the critical } \\
\text { sentence: } 0\end{array}$ \\
\hline Comparative & $\begin{array}{l}\text { There is a comparative expression in the critical } \\
\text { sentence: } 1 \text {, } \\
\text { There is not a comparative expression in the } \\
\text { critical sentence: } 0\end{array}$ \\
\hline Specific/Abstract & Specific:1, Neutral:0, Abstract:-1 \\
\hline Comments & Free text \\
\hline
\end{tabular}

Item 1) "Do you imagine senses of use of laptops if this critical sentence is showed?".

It is scored by 5 levels: "5: Very well", "4: well", "3: Neutral", "2: not very well", and "1: not at all".

Item 2) "Why can you imagine or why can you not imagine 
senses of use?".

It is answered by free text.

We set the scoring items from the result of the preliminary experiment. The characteristics of a critical sentence which were described frequently in the answers to Item 2 in the samples with high scores in Item 1 are derived as the scoring items in the main experiment.

TABLE III: THE METHODS OF DIVIDING SAMPLES FOR FACTOR ANALYSIS

\begin{tabular}{|c|c|c|c|}
\hline \multirow{2}{*}{\multicolumn{2}{|c|}{ samples examinees }} & \multirow{2}{*}{ All } & Usefulness \\
\hline & & & Scored by 4 or 5 \\
\hline \multicolumn{2}{|c|}{ All } & Analysis A & Analysis B \\
\hline \multirow[t]{2}{*}{ Important laptop's feature } & Speed of CPU & \multirow{2}{*}{ Analysis C } & \\
\hline & Portability & & \\
\hline
\end{tabular}

\section{2) Making samples}

We conducted the main experiment to make samples for analysis in Prodecure II. The examinees of the experiment were 18 male university students and 2 female students of 20 s. We describe the experiment in detail. Each of the examinee has assumed the scene where he/she buys the laptop "Toshiba dynabook satellite B451" in a specific online-shopping website. In below, "Toshiba dynabook satellite B451" is referred to as "dynabook". The examinee also assumed that he/she can obtain information of "dynabook" from only the specification table provided in the website. Therefore, in the assumption, the examinee cannot obtain information of "dynabook" from other source, for example, other websites or the real world. We presented the 137 critical sentences. Under this assumption, each of the examinee has scored the critical sentences extracted in all of presented "dynabook" reviews. The examinee has scored all of the scoring items given in Table I in scoring each critical sentence. He was also allowed to refer the specification table for "dynabook" we prepared in advance. In this experiment, we used all of the critical sentences of all reviews of "dynabook" in "Rakuten dataset" [6] provided by Rakuten ichiba [7]. The specification table was taken from kakaku.com [8]. Table II shows the answer choices corresponding to each scoring item in Table I. As the result, we could make 2740 samples.

\section{Procedure III: Extracting "Utility Factors" by Factor Analysis}

In Procedure III, we extract "utility factors". We use factor analysis on the samples made in Procedure II. If we can extract "utility factors", useful information can be provided from general reviews to customers in online-shopping by using "utility factors". However, reviews include a lot of information having nothing to relate with products. Therefore, it is desirable that only useful information is extracted by using "utility factors" with high accuracy. In this research, we execute factor analyses by 3 ways. The 3 ways are different in the viewpoint of the target samples. The 3 ways are referred to as Analysis A, Analysis B, and Analysis C. We extract the set of "utility factors" by each of the 3 factor analyses. In addition, we verify the accuracy of the set of "utility factors" extracted by each analysis to derive useful critical sentences with it. Table III shows the target samples of these analyses.

In Analysis A, we execute a factor analysis using all of the samples obtained in the experiment described in $b$ ). In Analysis B, we execute a factor analysis using the samples scored by 4 or 5 in the item "Usefulness". Analysis B extracts "utility factors" from only samples scored as to be useful. In
Analysis C, we execute factor analyses using each of 2 examinee clusters of the samples. To generate these clusters, we firstly classified the examinees into 2 groups according to their important points in buying laptops through the result of a questionnaire. In Analysis C, a factor analysis is executed on all of the samples generated by the examinees in each group. Therefore, Analysis C executes a factor analysis using each of sample clusters classified by the corresponding examinees' tastes. In Analysis C, we executed some questionnaires in order to classify the examinees from some viewpoint of tastes. However, we execute factor analyses using sample clusters divided from the viewpoint of laptops' features examinee think the most important. It is because the scores of samples differ among clusters most significantly in this viewpoint.

\section{FACTOR ANAYSES AND RESULTS FOR "UTILITY FACTORS" EXTRACTION}

In this section, we explain Analysis A, B, and C for Procedure III in detail. We also give their results and discussion about the derived "utility factors".

\section{A. Analysis A: Factor Analysis with All of the Samples}

In Analysis A, we firstly executed a factor analysis with all of the samples. The observed variables for each sample are the 8 scores for the scoring items given in Table I and Table II. Two factor analyses, preliminary and main ones, were processed to extract factors validly. The preliminary factor analysis used all of the observed variables. Referring its result, the main factor analysis was processed by removing inappropriate observed variables. A factor extracted by the main factor analysis is identified as a "utility factor" on critical sentences of product reviews. The set of the meaningful observable variables for each extracted factor, which have significant relationship with the factor, represents the characteristics of critical sentences that are useful from the point of view the corresponding "utility factor". In the preliminary factor analysis, we checked the factor loadings. A factor loading is the degree of relationship between an extracted factor by the factor analysis and each observable variable. The factor loading takes a value from -1 to 1 . As the result of checking the factor loadings, we found an observable variable whose absolute values of the factor loadings for all of the extracted factors are under 0.2. Such observable variable is considered not to have impact on the factors. Such observable variable was "Number". Therefore, we executed the main factor analysis by removing "Number" observable variable scores from the samples. As the result, 
we extracted 3 factors. We refer to these factors as the factors 1,2 , and 3 , in below of this subsection. For each of the 3 extracted factors, we could find the observable variables whose absolute values of the factor loadings for the factor are equal to or larger than 0.2 . Those observable variables were espoused as the meaningful observable variables for each of the 3 factors. We explain the meaningful observable variables for each of the 3 factors. The espoused observable variables for the factor 1 are "New knowledge" and "Specific/Abstract". Those for the factor 2 are "Structure of sentence" and "Specific/Abstract". Those for the factor 3 are "Adjective", "Subjective/Objective", "Positive/Negative", and "Comparative".

TABLE IV: ANALYSIS A: THE MEANINGFUl OBSERVABLE VARIABLES AND THEIR REPRESENTING CHARACTERISTICS OF EACH FACTOR ${ }^{1}$

\begin{tabular}{|c|c|c|}
\hline Factor 1 & Factor 2 & Factor 3 \\
\hline $\begin{array}{l}\text { There is a new knowledge } \\
\text { [New knowledge] } \\
\text { Specific } \\
\text { [Specific/Abstract] }\end{array}$ & $\begin{array}{l}\text { Very steady } \\
\text { [Structure of sentence] } \\
\text { Specific } \\
\text { [Specific/Abstract] }\end{array}$ & $\begin{array}{l}\text { There is an adjective except last of sentence } \\
\text { [Adjective] } \\
\text { Objective } \\
\text { [Subjective/Objective] } \\
\text { Negative } \\
\text { [Positive/Negative] } \\
\text { There is a comparative expression in the } \\
\text { critical sentence } \\
\text { [Comparative] }\end{array}$ \\
\hline
\end{tabular}

TABLE V: ANALYSIS B: THE MEANINGFUl OBSERVABLE VARIABLES AND THEIR REPRESENTING CHARACTERISTICS OF EACH FACTOR ${ }^{1}$

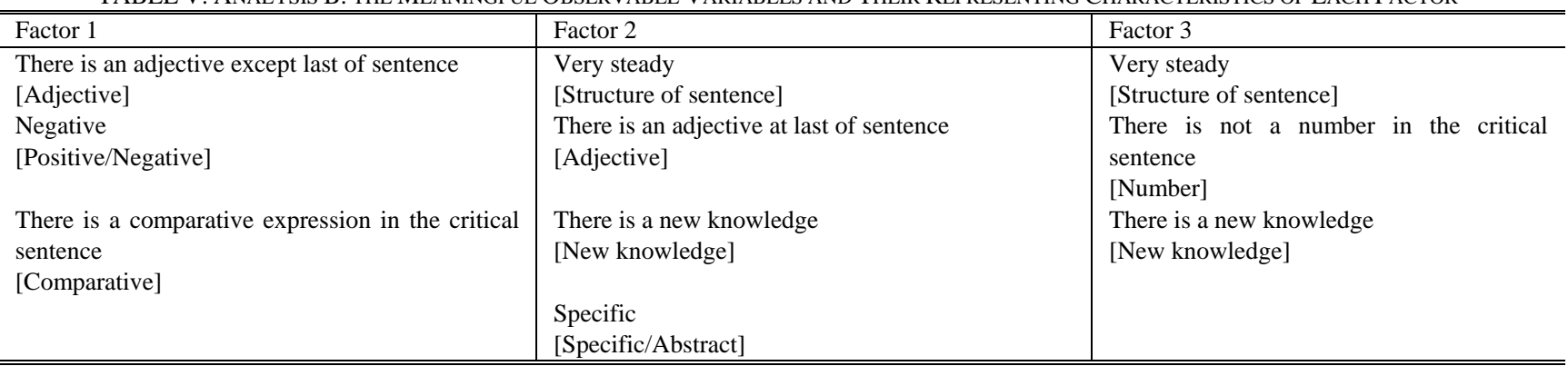

TABLE VI: “SPEED OF CPU” OF ANALYSIS C: THE MEANINGFUL OBSERVABLE VARIABLES AND THEIR REPRESENTING CHARACTERISTICS OF EACH FACTOR ${ }^{2}$

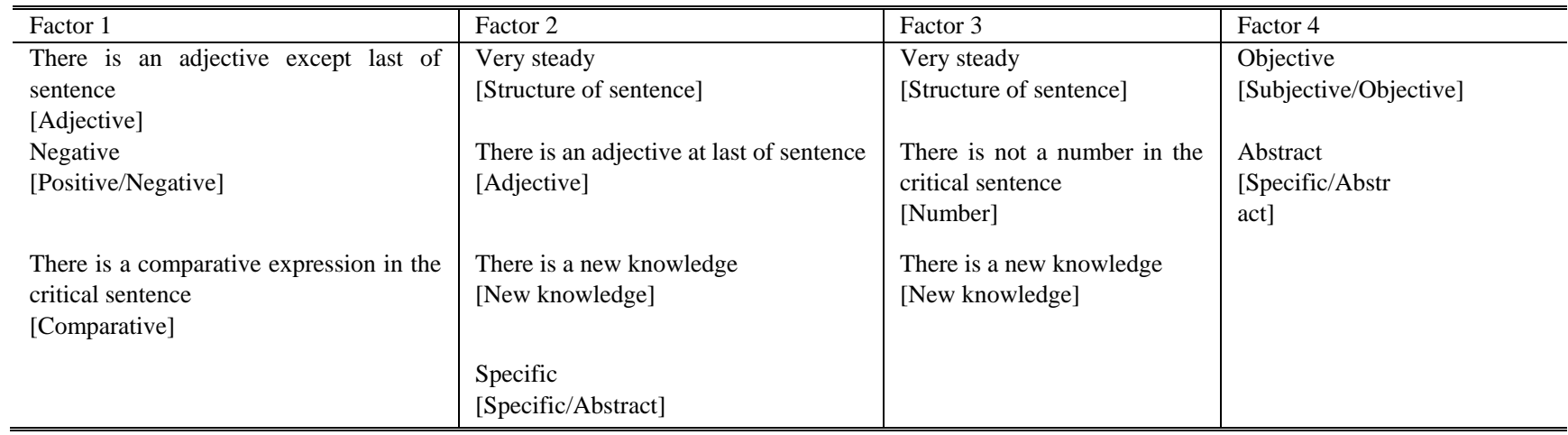

TABLE VII: "PORTABILITY” OF ANALYSIS C: THE MEANINGFUL OBSERVABLE VARIABLES AND THEIR REPRESENTING CHARACTERISTICS OF EACH FACTOR ${ }^{2}$

\begin{tabular}{|c|c|c|}
\hline Factor 1 & Factor 2 & Factor 3 \\
\hline $\begin{array}{l}\text { There is an adjective except last of sentence } \\
\text { [Adjective] } \\
\text { Negative } \\
\text { [Positive/Negative] } \\
\text { There is a new knowledge } \\
\text { [New knowledge] } \\
\text { There is a comparative expression in the } \\
\text { critical sentence } \\
\text { [Comparative] }\end{array}$ & $\begin{array}{l}\text { Very steady } \\
\text { [Structure of sentence] } \\
\text { There is an adjective at last of sentence } \\
\text { [Adjective] } \\
\text { There is a new knowledge } \\
\text { [New knowledge] } \\
\text { Specific } \\
\text { [Specific/Abstract] }\end{array}$ & $\begin{array}{l}\text { Very steady } \\
\text { [Structure of sentence] } \\
\text { Negative } \\
\text { [Positive/Negative] } \\
\text { There is not a number in the critical sentence } \\
\text { [Number] } \\
\text { There is a new knowledge } \\
\text { [New knowledge] }\end{array}$ \\
\hline
\end{tabular}

We checked the factor scores. A factor score is the weight of an extracted factor for a sample. We calculated the average of the factor scores for the samples with each level of "Usefulness" scoring item. We were able to find these facts. The factor scores in the factor 1 and the factor 2 increase as the "Usefulness" score increases. The factor score in the factor 3 decreases as the "Usefulness" score increases. Therefore, the factor 1 and the factor 2 have positive interrelationship with "Usefulness". The factor 3 has negative interrelationship with "Usefulness". Thus, guaranteeing high scores for the meaningful variables in each of the factors 1 and 2 are espoused as the characteristics of useful critical

\footnotetext{
${ }^{1}$ The element of sells is composed of characteristics [observable variable]

${ }^{2}$ The element of sells is composed of characteristics [observable variable]
} 
sentences from the point of the corresponding factor. In the factor 3, low scores for its meaningful variables are espoused as the corresponding characteristics. Table IV shows the characteristics of useful critical sentences derived from the meaningful observable variables for each of the 3 factors in Analysis A.

\section{B. Analysis B: Factor Analysis with All of Samples of High "Usefulness" Scores}

In Analysis B, we executed a factor analysis with the samples scored by 4 or 5 in the scoring item "Usefulness". Analysis B is performed by the preliminary and main factor analyses similar to Analysis A. In the preliminary analysis, we executed a factor analysis by the 8 observable variables same to those in Analysis A. After that, we found an observable variable whose absolute values of the factor loadings were under 0.2 for all of the extracted factors. This observable variable was "Subjective/Objective". Therefore, we removed "Subjective/Objective" values from the samples and we executed the main factor analysis. As the result, we extracted 3 factors, referred to as the factors 1, 2, and 3 in below in this subsection. For each of the 3 factors, there were the observable variables whose absolute values of the factor loadings for the factor is equal to or larger than 0.2. Those observable variables were espoused as the meaningful observable variables for the factor. However, there was only one observable variable whose absolute value of the factor loading for the factor 3 was equal to or larger than 0.2 . Therefore, the third factor extracted in the preliminary factor analysis in Analysis B was espoused instead of using the factor 3 directly. Thus, for the factor 3, the corresponding meaningful observable variables are derived from the factor obtained by the preliminary analysis. We explain the meaningful observable variables in each of the 3 factors. Those in the factor 1 are "Adjective", "Positive/Negative", and "Comparative". Those in the factor 2 are "Structure of sentence", "Adjective", "New knowledge" and "Specific/Abstract". Those in the factor 3 are "Structure of sentence", "Number", and "New knowledge".

The factors extracted in Analysis B are similar to the factors extracted in Analysis A. The factor 1 extracted in Analysis B is similar to the factor 3 extracted in Analysis A. The factor 2 and the factor 3 extracted in Analysis B is similar to the factor 1 and the factor 2 extracted in Analysis A, respectively. Therefore, low scores of the meaningful observable variables in the factor 1 were espoused as the characteristics of useful critical sentences similar to the factor 3 of Analysis A. High scores of the meaningful observable variables in the factor 2 and the factor 3 were espoused as the characteristics similar to the factors 2 and 3 of Analysis A. Table V shows the characteristics of useful critical sentences derived from the meaningful observable variables for each of the 3 factors in Analysis B.

\section{Analysis C: Factor Analysis with Samples Classified by Examinee's Taste}

In Analysis $\mathrm{C}$, we executed factor analyses with the samples by each cluster's examinee. These clusters were made according to examinees' tastes by a questionnaire about a laptop's feature each examinee thinks the most important. For the samples of each cluster's examinee, preliminary and main factor analyses were performed similar to Analysis A. The set of "utility factors" can be extracted in each cluster. In this questionnaire, we were able to classify the examinees into 3 clusters. The 3 clusters were the examinees thinking speed of CPU is the most important for laptops, the examinees thinking portability is the most important for laptops, and the examinees thinking price is the most important for laptops. In this paper, these 3 clusters are referred to as "Speed of CPU", "Portability", and "Price". We firstly selected target clusters from these 3 clusters. A factor analysis similar to Analysis A was performed in the samples of each of the clusters. We calculated the average of the factor scores of the samples with each level of "Usefulness" for every cluster. This process shows the relationship between factor score and "Usefulness" score in each cluster. We have checked the interrelationship between the averages of the factor scores by 5 levels of "Usefulness" and "Usefulness" scores in every cluster. Then, there were interrelationship in "Speed of CPU" and "Portability" clusters while there was no interrelationship in "Price" cluster. Therefore, we executed preliminary factor analyses for "Speed of CPU" and "Portability".

After that, there were the observable variables whose absolute values of the factor loadings are under 0.2 for all of extracted factors for every target cluster. Therefore, we removed these observable variables and we executed the main factor analysis again in every target cluster. As the result, the factor analyses extracted 4 factors in "Speed of CPU" and extracted 3 factors in "Portability". There were the observable variables whose absolute values of the factor loadings are equal to or larger than 0.2 for each extracted factor. Those observable variables were espoused as the meaningful observable variables for each factor. Thus, the meaningful observable variables in the factors of every cluster were espoused according to the interrelationship between the averages of the factor scores for 5 levels of "Usefulness" and "Usefulness" scores in every cluster. Table VI shows the characteristics of useful critical sentences derived from the meaningful observable variables for each of the 4 factors of "Speed of CPU" cluster. Table VII does those for each of the 3 factors of "Portability" cluster.

TABLE VIII: EFFECTIVENESS EVALUATIONS OF "UTILITY FACTORS" BY ANALYSIS

\begin{tabular}{l|r|r|r|r}
\hline \hline & Analysis A & Analysis B & $\begin{array}{l}\text { Analysis C } \\
\text { "Speed of } \\
\text { CPU" }\end{array}$ & $\begin{array}{l}\text { Analysis C } \\
\text { "Portability" }\end{array}$ \\
\hline Precision(\%) & 0.359 & 0.486 & 0.511 & 0.512 \\
\hline \hline
\end{tabular}

\section{EFFECTIVENESS EVALUATIONS OF "UTILITY FACTORS"}

The factors extracted by Analysis A, Analysis B, and Analysis $\mathrm{C}$ are sets of "utility factors" of reviews. In this section, we evaluate usefulness of the factors extracted by each of Analysis A, Analysis B, and Analysis C as "utility factors" of reviews. We evaluate usefulness by checking how accurately the "utility factors" can extract useful information in reviews. In this section, we say that a sample has a "utility factor" if the sample's scores satisfy all of the characteristics of critical sentences on the "utility factor" given in Table IV, 
Table V, Table VI, or Table VII. We define the samples whose usefulness is scored by 4 or 5 by the examinees as the correct data. We define the sample having all "utility factors" extracted by each of Analysis A, Analysis B, and Analysis C as the extracted data for the "utility factors". We also define a sample belonging to the correct or extracted data as a correct or extracted sample, respectively. We can say the "utility factors" extracted by Analysis A have high effectiveness when these "utility factors" extracted data matches the correct data significantly. We can say the same thing in Analysis B and Analysis C. On these definitions, we calculate the precisions on Analysis A, Analysis B, and Analysis C by using (1).

$$
\text { Precision }=\frac{\text { Number of extracted samples in correct data }}{\text { Number of extracted samples }}
$$

We consider precision is important to delete not useful information as much as possible, because reviews have noise in bulk. Table VIII shows the precisions of each "utility factors" extracted by the analyses. In this result, we can understand precision is higher by using the samples classified finely. Therefore, we can say factors extracted by a factor analysis using samples classified finely have high precision. In Analysis C, we could not extract factors successfully in cluster "Price". It may be because customers who emphasize prices can easily judge products are good or bad by checking products' specification table on website. We consider such customers judge products are good or bad from the viewpoint of the products' features which they emphasize secondly such as functions and strong points when the prices are not good for the customers. Therefore, we consider the scores vary in wide range among the examinees because the examinee's secondly important features of products differ in each other.

\section{CONCLUSION}

In this paper, we discovered common factors between useful reviews for deciding products customers buy, called "utility factors", in online-shopping by analyzing reviews. The flow to find the common factors from actual reviews has 3 steps. First, we extracted critical sentences evaluating products in reviews written on an online-shopping website. Second, these critical sentences were given values to evaluate their characteristics by examinees for factor analyses on an experiment. Finally, we did 3 kinds of factor analyses using these values, which are Analysis A, B, and C. These analyses differ by the degrees and target customers on usefulness of the target critical sentences. "utility factors" were extracted in each of these 3 analyses. As the result, the "utility factors" extracted by Analysis $\mathrm{C}$ suggested the highest precision in these 3 analyses. This precision means the "utility factors" can extract useful information from reviews with high accuracy. Analysis $\mathrm{C}$ targeted only the values of the critical sentences which are useful for the examinees having the same specific taste. The precision for "utility factors" are improved by using critical sentences which attracts more specific customers and executing factor analyses by such critical sentences.

We will find the algorithms to extract useful information from reviews by using the "utility factors" extracted in this research.

\section{REFERENCES}

[1] S. Huang, D. Shen, W. Feng, C. Baudin, and Y. Zhang, "Improving product review search experiences on general search engines," in Proc. 11th International Conference on Electronic Commerce, 2009, pp 107-116.

[2] D. Okanohara and J. Tsujii, "Assigning Polarity Scores to Reviews Using Machine Learning Techniques", in Proc. Natural Language Processing, 2005, pp. 314-325.

[3] N. Kobayashi, K. Inui, Y. Matsumoto, K. Tateishi, and T. Fukushima, "Collecting Evaluative Expressions for Opinion Extraction", in Proc. Natural Language Processing, 2004, pp. 596-605.

[4] A. Ghose and P. G. Ipeirotis, "Estimating the Helpfulness and Economic Impact of Product Reviews: Mining Text and Reviewer Characteristics," IEEE Transaction on Knowledge And Data Engineering, vol. 23, no. 10, pp. 1498-1511, 2004.

[5] Mecab. [Online]. Available: http://mecab.googlecode.com/svn/trunk/mecab/doc/index.html

[6] Rakuten dataset. [Online]. Available: http://rit.rakuten.co.jp/rdr/index.html

[7] Rakuten ichiba. [Online]. Available: http://www.rakuten.co.jp/

[8] Kakaku.com. [Online]. Available: http://kakaku.com/

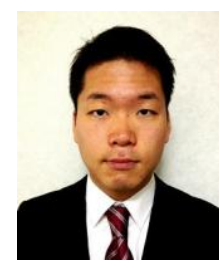

Kenjo Yasui was received B.E from Ritsumeikan University in 2013. He advanced Graduate School of Ritsumeikan University. He engages in the research on data engineering. He is member of IPSJ.

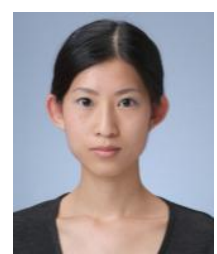

Fumiko Harada was received B.E. and M.E, and Ph.D degrees from Osaka University in 2003, 2004, and 2007, respectively. She joined Ritsumeikan University as an assistant professor in Ritsumeikan University in 2007 , and is currently a lecturer. She engages in the research on real-time systems and data engineering. She is a member of IEEE.

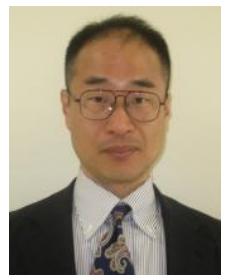

Hiromitsu Shimakawa was received Ph.D degree from Kyoto Univ. in 1999. He joined Ritsumeikan Univ. in 2002. Currently, he is a professor in Ritsumeikan Univ. His research interests include data engineering, usability, and integration of psychology with IT. He is a member of IEEE and ACM. 